\title{
Dynamic features creating (which cause) the blood direction inside the left ventricle
}

\author{
Mersedeh Karvandi ${ }^{2,}$, Saeed Ranjbar ${ }^{1}$, Seyed Ahmad Hassantash ${ }^{1}$ \\ ${ }^{1}$ Modarres Hospital, Institute of Cardiovascular Research, Shahid Beheshti University of Medical Sciences, Tehran, Iran \\ ${ }^{2}$ Taleghani Hospital, Shahid Beheshti University of Medical Sciences, Tehran, Iran
}

\section{Email address:}

mersedeh_karvandi@ipm.ir (M. Karvandi), sranjbar@ipm.ir(S. Ranjbar), sahassan@pol.net (S. A. Hassantash)

\section{To cite this article:}

Mersedeh Karvandi, Saeed Ranjbar, Seyed Ahmad Hassantash. Dynamic Features Creating (Which Cause) the Blood Direction Inside the Left Ventricle. International Journal of Medical Imaging. Vol. 2, No. 2, 2014, pp. 14-18. doi: 10.11648/j.ijmi.20140202.12

\begin{abstract}
Background: Demonstration of blood flow direction inside cardiac chambers can provide valuable information in normal subjects and pathologic cardiac processes. In our center we have already created a software able to generate the left ventricle (LV) model, the most important chamber of the heart. Method: The fundamental quantity that describes the motion of the fluid is represented by the vorticity defined as the tendency of fluid elements to spin; more precisely, vorticity can be related to rotation of fluid elements and the formation of circulatory areas. Quantitative parameters of the intraventricular vortex were also extracted on the basis of the vorticity. Result: In the left ventricle: blood flows through the mitral valve, hits the lateral left ventricular free wall, turbulences clockwise at the apex of the heart, hits the septal wall and flows towards the aortic valve. Conclusion: Technical points in our field of study can succeed us to gain a good understanding about the material. For blood flow direction inside the left ventricle, we can propose some tricky points:

1- $\quad$ Factors affecting blood direction inside the left ventricle?

2- Why does the blood flow at the present direction inside the left ventricle?
\end{abstract}

Keywords: Mathematical Modeling, Blood Flow Direction, The Left Ventricular Modeling, Wall-Fluid Interaction Problem

\section{Introduction}

Mathematical boundary method is a new technique of determining the velocity and the direction of fluid streams by analyzing the change in position of small particles that drift with the fluid. With the recent development of echocardiographic technology, it is now possible to apply this approach to echocardiographic imaging. 1

The growing knowledge about the structure and function of the ventricle was of high interest to us in the context of ventricular vortex development in the Normal subject. A vortex is a mass of fluid with a whirling or circular motion, thus containing kinetic energy. It is supposed to increase cardiac efficiency by maintaining the momentum of the inflowing blood in diastole and, thus, facilitating systolic ejection of blood into the left ventricular outflow tract. The demonstration of diastolic vortex formation in normal human hearts and their distortion by valve surgery led to our study objective to what extent blood flow patterns in congenitally abnormal, functionally univentricular hearts of patients are different from normal ones. Left ventricular
(LV) fluid dynamics represent an elegant flow phenomenon, starting with the trans-mitral jet reaching a maximal velocity of about $100 \mathrm{~cm} / \mathrm{s}$ in traveling a distance as short as only a few centimeters. This volume of blood must then turn around to exit the left ventricle via the aortic valve within a fraction of a second while maintaining its initial high velocity. For this to happen properly, nature has chosen a vortex flow (a swirling motion of fluid), which is developed during diastole, to redirect the trans-mitral flow toward the LV outflow tract. This track of motion guarantees efficient transfer of the fluid momentum and minimizes the ventricular energy dissipation Despite improvements in intra-ventricular flow visualization by means of advanced noninvasive imaging modalities, experimental and numerical models, several aspects of the corresponding fluid dynamics phenomena are still unclear. The key reason for the lack of quantitative information concerning cardiac flow is the lack of technology that can reliably map the spatial and temporal details of the flow while being feasible in routine clinical use. Color Doppler imaging, as a tool to obtain intra-cardiac flow information, considers only a single component of blood velocity along 
the transducer scan line and does not provide quantitative information with respect to vortex formation, recirculation areas, and flow stagnation zones, Because of these limitations, color Doppler imaging may not provide adequate information for either the reconstruction of velocity vector fields or the actual direction of streamlines. Several attempts have been made to incorporate quantitative fluid dynamics into echocardiography using particle-tracking algorithms. This study aimed at assessing vortex flow patterns in Normal Subjects circulation in comparison with the other technology. ${ }^{2-13}$

\section{Method}

\subsection{Left Ventricular Modeling of the Myocardium}

Ranjbar S. et al. (2013) ${ }^{20}$ recently developed the first novel left ventricular myocardial model mathematically based on echocardiography, by MATLAB software and LSDYNA software in normal subjects, which dynamic orientation contraction (through the cardiac cycle) of every individual myocardial fiber could be created by adding together the sequential steps of the multiple fragmented sectors of that fiber. The left ventricular myocardial modeling of the heart shows that in normal cases myocardial fibers initiate from the posterior-basal region of the heart, continues through the left ventricular free wall, reaches the septum, loops around the apex, ascends, and ends at the superior-anterior edge of left ventricle (Figure 1).

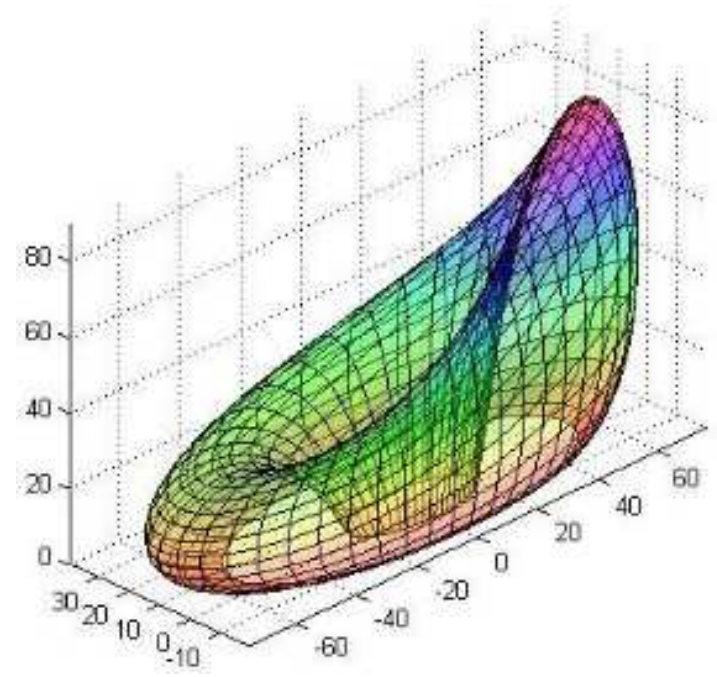

Figure 1. Impact of the direction of blood flow path to the left ventricular torsional deformation.

\subsection{Achievability of the Assessment of the Mitral Valve Leaflets by Mathematical Equations of Inelasticity}

The mechanics of the mitral valve leaflet as a nonlinear, inelastic and anisotropic soft tissue results from an integrated response of many mathematical/physical indexes' that illustrate the tissue. In the past decade, finite element modeling of complete heart valves has greatly aided evaluation of heart valve surgery, design of bioprosthetic valve replacements, and general understanding of healthy and abnormal cardiac function. Such a model must be based on an accurate description of the mechanical behavior of the valve material. It is essential to calculate velocity/displacement and strain rate/strain at a component level, that is to work at the cellular level. In this study we developed the first three-dimensional displacement vectors field in the characterization of mitral valve leaflets in continuum equations of inelasticity framework based on echocardiography. Echocardiography imaging of the mitral valve motion during the cardiac cycle of planes TTE in the short axis, long axis and four chamber views, and multiplanes TEE in the lower esophageal views at $0,10,15,30$, $45-60,75,80,90,110-125$ and 135 degrees were prospectively acquired for 200 healthy subjects of 65 timeframes from diastole to systole(early diastole, mid diastole, atrial systole, end of diastole, end of systole). In each plane and for each frame during diastole to systole, mitral valve annulus and leaflets, and bases, middles and tips of AML and PML, were manually identified using MATLAB software. 3D MV annulus geometry, leaflets measurements, AML and PML positions were automatically computed for each frame, and used as input to displacement vectors modeling of the mitral valve leaflets. By solving mathematical equations of inelastic properties of leaflets, each plane was replaced with 60 vectors of displacement and ultimately the mitral valve leaflets were realized by 1125 vectors of displacements where show also translations, rotations and pure strains of bases, middles and tips of AML and PML simultaneously per cardiac cycle. Preliminary results concerning a different aspect of MVL biomechanics, such as leaflets dynamics, displacements/velocities and strain rates/strains of points on leaflets, were in good agreement with in echocardiographic observations $^{14}$ (Figures 2 and 3).

A

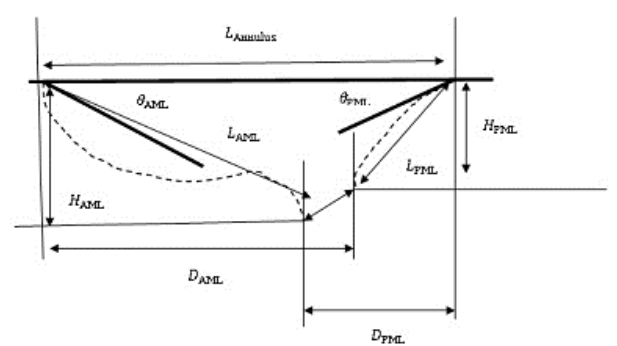

B

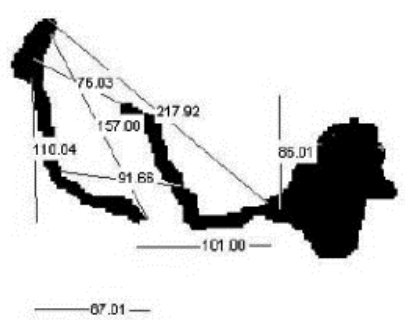

Figure 2. Parameters used to define the geometry of Mitral valve. B) The numerical calculation of geometric parameters of a mitral valve in MATLAB software. 


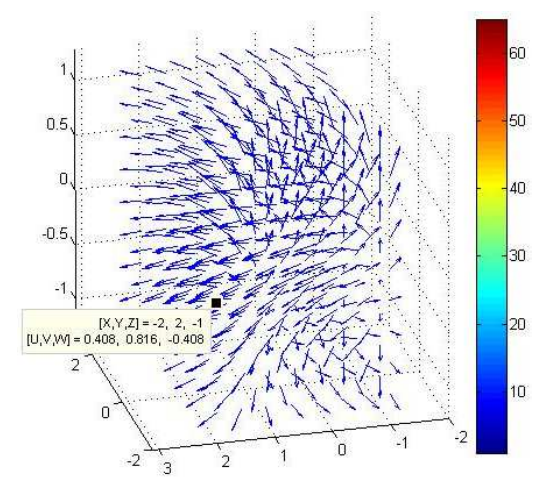

Figure 3. Velocity vector field applied on mitral valve leaflets.

The medical ethics committee of Shahid Beheshti Medical University approved this study. All procedures were in accordance with the Helsinki declaration, and no harm was experienced by the participants.

\subsection{Fluid Dynamic Inside the Left Ventricle by Using 1, 2 in above}

The standard way to model the motion of blood inside the left ventricle would be to treat the left ventricle as an elastic membrane obeying Newton's laws of motion with forces calculated in part from the elasticity of the membrane and in part by evaluating the fluid stress tensor on the surface of the membrane. Then the fluid equations would have to be supplemented by the constraint that the velocity of the fluid on either side of the membrane must agree with the instantaneously known velocity of the elastic membrane itself. There is a difficulty with this standard approach to the problem. Challenge is the practical one of evaluating the fluid stress tensor on either side of the boundary. This seems difficult (or at least messy) to do numerically, unless the computational grid is aligned with the boundary. On the other hand, in a moving boundary problem, it is both expensive and complicated to recompute the grid at every time step in order to achieve alignment. This means that the sum of the elastic force and the fluid force on any part of the boundary has to be zero. Once we know this, it becomes unnecessary to evaluate the fluid stress tensor at the boundary at all! We can find the force of any part of the boundary on the fluid by evaluating the elastic force on that part of the boundary. (Note the use of Newton's third law: the force of boundary on fluid is minus the force of fluid on boundary) All we need is a method for transferring the elastic force from the boundary to the fluid. On a Cartesian grid, this may be done by spreading each element of the boundary force out over nearby grid points. The particular way that this is done in the boundary method involves a carefully constructed approximation to the Dirac delta function. This forcespreading operation defines a field of force on the Cartesian lattice that is used for the fluid computation. Then the fluid velocity is updated under the influence of that force field. The Navier-Stokes solver that updates the fluid velocity does not know about the any consideration of the heart left ventricle geometry; it just works with a force field that happens to be zero everywhere except in the immediate of the vorticity region. This approach can be used for instructional purposes and diagnosis of heart ailments.

The local fluid velocity at a point of the elastic boundary is evaluated by interpolation from the Cartesian grid. The same approximate delta function that was used to spread force can also be used to get an interpolation operator that is the adjoint (or transpose) of the force-spreading operator. In summary, the boundary method avoids many of the difficulties and pitfalls of the standard approach to fluidstructure interaction. By representing an elastic boundary in terms of the forces applied by the immersed elastic boundary to the fluid, the boundary method avoids any assumption of boundary geometry in the fluid computation; makes it unnecessary to evaluate the fluid stress tensor at the elastic boundary; and makes it possible to simulate elastic boundaries, like the chambers of the human heart.

\section{Result}

During ejection, the direction of the flow was from LV apex to LV out flow track (LVOT). After the aortic valve closure, in the early isovolumic relaxation (IVR) period, the direction of flow reversed from LV base to apex with a brief appearance of vortex in the proximity of the mitral valve. During the mid-late IVR period, the non-vertical columnar flow was seen directed from base to apex. In the early diastolic period, an irrotational flow associated with early LV filling dominated the vector representation of flow. In diastasis, a relatively apically located vortex was seen. This was followed by a late filling phase of atrial contraction, which was characterized by irrotational flow obscuring the vortex. After mitral valve closure, in the early isovolumic contraction (IVC) period, the vortex was relocated in the proximity of anterior mitral leaflet in the LVOT region and directed flow from LV apex to LVOT. During late IVC period, the vortex persisted in the LVOT region and directed flow towards the aortic valve. With the aortic valve opening and ejection, the vortex dissipated with continued flow from apex to LVOT(Figure 4). ${ }^{15}$

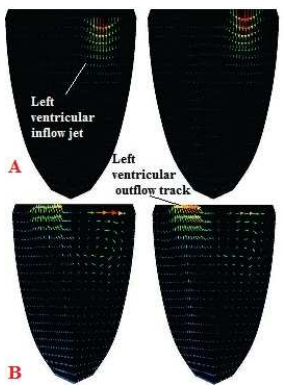

Figure 4. In the late filling phase that was characterized by an irrotational flow obscuring the vortex This was followed by in the early isovolumic contraction (IVC) period (A), the vortex was relocated in the proximity of the anterior mitral leaflet in the LVOT region (B). During the late IVC period, the vortex persisted in the left ventricular outflow tract region and directed flow towards aortic valve. 


\section{Discussion}

The insights into LV vortex flow may have additional and potentially incremental value over the conventional methods to assess LV function. Vortex flow may influence stroke output and efficiency of the LV by redirection of intra-ventricular flow. Diastolic LV vortex characterization may have implication for diastolic volumetric filling and may provide an index that links diastolic filling to systolic stroke volume. There are other potential applications such as association in LV vortex to dyspnea and relationship to symptoms in patients with heart failure.

In patients with Dilated Cardiomyopathy (DCM), the vortex was located at the center of the LV throughout diastole and systole and did not redirect flow in a coherent, sequential fashion as in normal subjects. In normal subjects, the average vortex was compact, elliptically.

Shaped, and was located apically. This vortex was persistent during diastole and directs vectors towards the
LVOT. In patients with DCM, a spherical, centrally located vortex was observed with incoherent direction of $\mathrm{LV}$ flow. ${ }^{16-18}$

As demonstrated in the flow direction inside the LV is completely different from each other when the two perpendicular valve directions are chosen as much as when compared with the normal heart. In a normal LV Initial pressure force is impacted on the free wall in the contrary to when an anatomically directed prosthetic mitral valve (PMV) is performed with initial pressure impacted to LV inter-septum. And flow Patterns of PMV's in Anatomic Orientation that All patients had their prosthetic mitral valve prostheses implanted in anatomic orientation (i.e. with the hinge positions approximating commissures of the previous native valve) The 2 outer orifices generate jets directed toward the left ventricular septum wall. In general, the resulting vorticity pattern in hearts of patients with anatomically replaced in PMV is opposite to that seen in healthy hearts(Figure 5). ${ }^{19,20}$

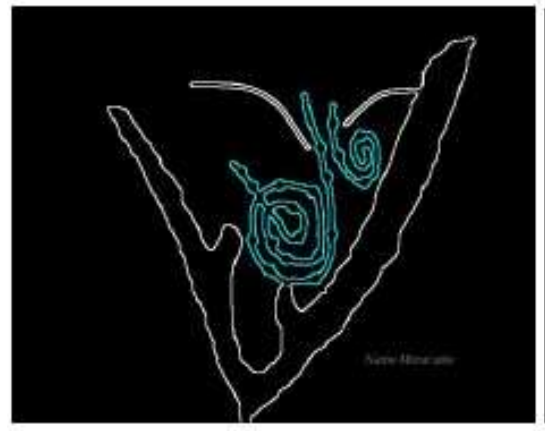

Native mitral valve

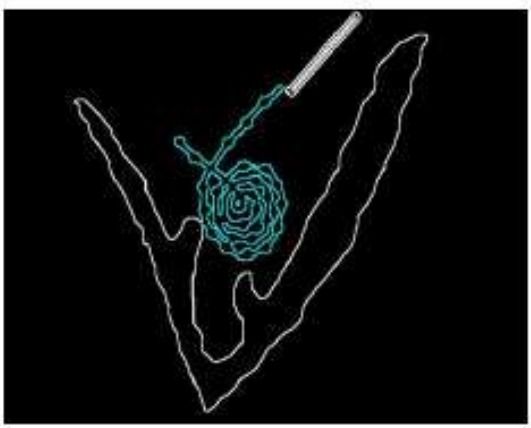

Anatomical prosthetic MVR

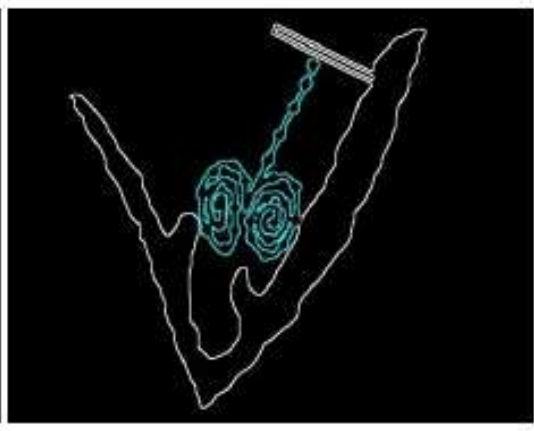

Anti-anatomical prosthetic MVR

Figure 5. Impact of prosthetic mitral valve direction on blood flow path inside the left ventricle.

\section{Conclusion}

As stated by Jacques Hadmard in 'science and hypothesis', mathematical creation and mathematical intelligence are not without connection with creation in general and with general intelligence. A great proportion of prominent mathematicians have been creators in other fields. One of the greatest, Gauss, carried out important and classical experiments on magnetism; and Newton's fundamental discoveries in optics are well known. And Leibniz influenced by their mathematical abilities on philosophical ones. The researches we propose here, are motivated by our background and previous research, and are supposed to indicate our general directions in forthcoming research. Following Poincare, each research subject is formed by an attempt to link two fields in sciences, in order to contribute to at least one of the two. Always the medical part is the part which takes the benefit.

\section{Conflict of Interest}

There is no conflict of interest.

\section{Supplementary Files}

\section{US Patents}

1-System and method for modeling left ventricle of heart Patent Number: 8,414,490

Issue Date of Patent: 04-09-2013

www.google.com/patents/US20110172539US Pat. App Filed 14 Mar 2011 - Mersedeh Karvandi 600/453 Left Ventricle Of Heart (57) Abstract (76) Iiiveiiiois: Saeed Ranjbar' Teiiran The embodiments herein provide a method and a system.

2- Solution Navier-stocks equations of the blood as a nonNewtonian fluid in the left ventricle.

Notice of Allowance, Date of issue: 01127/2014.

www.google.com/patents/US20110166467US Pat. App Filed 14 Mar 2011 - Mersedeh Karvandi,Saeed Ranjbar (Mersedeh Karvandi<19) United States US 20110166467A1 <12) Patent Application Publication <10) Pub. No.

3-Method for determining heat boundary value conditions of red blood cells in the neighborhood of myocardium. 
www.google.com/patents/US20110161013US Pat. App Filed 14 Mar 2011 - Mersedeh Karvandi Saeed Ranjbar (Mersedeh Karvandi<19) United States <12) Patent Application Publication <10) Pub. No.: US 2011/0161013 A1 US.

\section{References}

[1] Mittal R., Iaccarino G.: Immersed boundary methods.Annual Review of Fluid Mechanics.2005; 37:239261

[2] Kim WY., Walker PG., Pedersen EM., Poulsen JK., Oyre S., Houlind K.: Left ventricular blood flow patterns in normal subjects: a quantitative analysis by three-dimensional magnetic resonance velocity mapping. J Am SocEchocardiogr. 1995;26: 24-38

[3] Kilner PJ., Yang G-Z., Wilkes AJ., Mohiaddin RH., Firmin DN., Yacoub MH.: Asymmetric redirection of flow through the heart. Nature. 2000;404:59-61

[4] Bellhouse BJ.: Fluid mechanics of a model mitral valve and left ventricle. Cardiovasc Res.1972; 6:199-210

[5] Domenichini F., Pedrizzetti G., BaccaniB.:Threedimensional filling flow into a model left ventricle. J Fluid Mech. 2005;539:79-98

[6] Kim HB., Hertzberg JR., Shandas R.: Development and validation of echo PIV. Exp Fluids 2004; 36: 55-62

[7] Zheng H., Liu L., Williams L., Hertzberg JR., Lanning C., Shandas R.: Real time multicomponent echo particle image velocimetry technique for opaque flow imaging. ApplPhysLett 2006;88:1-3

[8] Sengupta PP., Khandheria BK., Korinek J., Jahangir A., Yoshifuku S., Miloseve I.: Left ventricular isovolumic flow sequence during sinus and paced rhythms: new insights from use of high-resolution Doppler and ultrasonic digital particle imaging velocimetry. J Am CollCardiol. 2007;49:899-908

[9] Hong G-R., Pedrizzetti G., Tonti G., Li P., Wei Z., Kim JK., et al.: Characterization and quantification of vortex flow in the human left ventricle by contrast echocardiography using vector particle image velocimetry. J Am CollCardiolImg.2008; 1:5-17
[10] Adrian RJ.:Particle-imaging techniques for experimental fluid mechanics. Ann Rev Fluid Mech. 1991 ; 23:261-304

[11] Kheradvar A., Kasalko J., Johnson D., Gharib M.: An in vitro study of changing profile heights in mitral bioprostheses and their influence on flow. ASAIO J. 2006; $52: 34-8$

[12] Batchelor GK.: An introduction to fluid dynamics. Cambridge, UK: Cambridge

[13] University Press 1970.Sengupta PP., Korinek J., Belohlavek M., Narula J., Vannan MA., Jahangir A.: Left ventricular structure and function: basic science for cardiac imaging. J Am CollCardiol.2006; 48:1988-2001

[14] Karvandi M., Ranjbar S., Hassantash S. A.: Achievability of the Assessment of the Mitral Valve Leaflets by Mathematical Equations of Inelasticity Based on EchocardiographyPoster presentation at AmericanEcho. Abstract: P2-92 2012.

[15] Geu-Ru Hong G. R., Pedrizzetti G., Giovanni Tonti G., Li P., Wei Z., Kim J.K., Baweja A., Liu S., Namsik Chung N., Houle H., Narul J., Vannan M. A.: Characterization and quantification of Vortex Flow in the Human Left Ventricle by contrast echocardiography using vector particle Image velocimetry. Jacc: Cardiovascular imaging.2008; 1:705-717

[16] Ranjbar S., Karvandi M., Hassantash S.A. :A novel mathematical based software for modeling the left ventricular myocardium. Poster presentation at Euroecho. Abstract: P448 2012.

[17] Ranjbar S., Karvandi M., Hassantash S. A.:A novel mathematical based software to demonstrate flow direction and curves inside the left ventricular myocardium.Poster presentation at Euroecho. Abstract: P142 2012.

[18] Ranjbar S., Karvandi M., Hassantash S. A.: A Novel Mathematical Fluid Dynamic of Left Ventricular Dilated Cardiomyopathy. Poster presentation at Americanecho. Abstract: P2-89 2012.

[19] Ranjbar S., Karvandi M., Hassantash S. A.: Impact of prosthetic mitral valve direction on blood flow path inside the left ventricle. . Poster presentation at Euroecho. Abstract: P516 2012.

[20] Ranjbar S., Karvandi M., Ajzachi M.: System and method modeling left ventricle of heart. US Patent 2013; patent number $8,414,490$. 